\title{
Boundedness of Oscillatory Hyper-Hilbert Transform along Curves on Sobolev Spaces
}

\author{
Jun $\mathrm{Li}^{1}$ and Guilian Gao ${ }^{2}$ \\ ${ }^{1}$ Department of Mathematics, Zhejiang University, Hangzhou 310027, China \\ ${ }^{2}$ School of Science, Hangzhou Dianzi University, Hangzhou 310018, China
}

Correspondence should be addressed to Guilian Gao; gaoguilian305@163.com

Received 1 March 2014; Accepted 6 May 2014; Published 19 May 2014

Academic Editor: Yongsheng S. Han

Copyright (C) $2014 \mathrm{~J}$. Li and G. Gao. This is an open access article distributed under the Creative Commons Attribution License, which permits unrestricted use, distribution, and reproduction in any medium, provided the original work is properly cited.

The oscillatory hyper-Hilbert transform along curves is of the following form: $H_{n, \alpha, \beta} f(x)=\int_{0}^{1} f(x-\Gamma(t)) e^{i t^{-\beta}} t^{-1-\alpha} \mathrm{d} t$, where $\alpha \geq 0$, $\beta \geq 0$, and $\Gamma(t)=\left(t^{p_{1}}, t^{p_{2}}, \ldots, t^{p_{n}}\right)$. The study on this operator is motivated by the hyper-Hilbert transform and the strongly singular integrals. The $L^{p}$ bounds for $H_{n, \alpha, \beta}$ have been given by Chen et al. (2008 and 2010). In this paper, for some $\alpha, \beta$, and $p$, the boundedness of $H_{n, \alpha, \beta}$ on Sobolev spaces $L_{s}^{p}\left(\mathbb{R}^{n}\right)$ and the boundedness of this operator from $L_{s}^{2}\left(\mathbb{R}^{n}\right)$ to $L^{2}\left(\mathbb{R}^{n}\right)$ are obtained.

\section{Introduction}

In the paper, we mainly discuss singular integrals in the following form:

$$
H_{n, \alpha, \beta} f(x)=\int_{0}^{1} f(x-\Gamma(t)) e^{i t^{-\beta}} t^{-1-\alpha} \mathrm{d} t,
$$

where $\alpha \geq 0, \beta \geq 0$, and $\Gamma(t)=\left(t^{p_{1}}, t^{p_{2}}, \ldots, t^{p_{n}}\right)$ denotes a curve in the $n$-dimensional spaces.

Operators of this kind originate from the significant Hilbert transform:

$$
H f(x)=p \cdot v \cdot \int_{\mathbb{R}} \frac{f(x-t)}{t} \mathrm{~d} t .
$$

In [1], Calderón and Zygmund brought in the rotation method, shifting the study of the homogeneous singular integral operators to that of directional Hilbert transforms:

$$
\begin{aligned}
T_{\Omega}(f)(x) & =p . v \cdot \int_{\mathbb{R}^{n}} f(x-y) \frac{\Omega(y /|y|)}{|y|^{n}} \mathrm{~d} y \\
& =\frac{1}{2} \int_{S^{n-1}} \Omega\left(y^{\prime}\right) H_{y^{\prime}}(f)(x) \mathrm{d} \sigma\left(y^{\prime}\right),
\end{aligned}
$$

where $\Omega$ is odd, and the directional Hilbert transform is

$$
H_{y^{\prime}}(f)(x)=\text { p.v. } \int_{\mathbb{R}} f\left(x-t y^{\prime}\right) \frac{\mathrm{d} t}{t} .
$$

In order to generalize the rotation method, Fabes and Rivière [2] introduced the Hilbert transform along curves:

$$
H_{\Gamma} f(x)=p \cdot v \cdot \int_{-\infty}^{+\infty} f(x-\Gamma(t)) \frac{\mathrm{d} t}{t} .
$$

Afterwards, the research of $H_{\Gamma} f(x)$ attracted many scholars, among which Wainger and his fellows contributed to it quite remarkably.

Another development derived from Hilbert transform is hypersingular Hilbert transforms:

$$
H_{\alpha} f(x)=p . v \cdot \int_{-1}^{1} f(x-t) \frac{\mathrm{d} t}{t|t|^{\alpha}}, \quad 0<\alpha<1 .
$$

As such operator has more singularity, $f$ is required to have some smoothness. It can be proved that $H_{\alpha}$ is bounded from $L_{\alpha}^{p}\left(\mathbb{R}^{n}\right)$ to $L^{p}\left(\mathbb{R}^{n}\right)$, where $1<p<\infty$.

A natural question is how to balance the more singularity due to $|t|^{\alpha}$, without extra smoothness of $f$. Since Hilbert transform is essentially "oscillatory," we can bring in an oscillatory factor $e^{i t^{-\beta}}$ in $H_{\alpha}$. So is the oscillatory hypersingular integral along curves in the following form:

$$
H_{n, \alpha, \beta} f(x)=\int_{-1}^{1} f(x-\Gamma(t)) e^{i|t|^{-\beta}} \frac{\mathrm{d} t}{t|t|^{\alpha}},
$$


where $\alpha \geq 0, \beta \geq 0$, and $\Gamma(t)=\left(t^{p_{1}}, t^{p_{2}}, \ldots, t^{p_{n}}\right)$ denotes a curve in the $n$-dimensional spaces.

In this direction, the thesis of Zielinski [3] was pioneering. In the case $n=2, \Gamma(t)=\left(t, t^{2}\right)$, he proved

$$
\left\|H_{2, \alpha, \beta} f\right\|_{L^{2}\left(\mathbb{R}^{2}\right)} \preceq\|f\|_{L^{2}\left(\mathbb{R}^{2}\right)} \Longleftrightarrow \beta \geq 3 \alpha .
$$

Later on, Chandarana [4] generalized the result of Zielinski into more common curves, showing the corresponding boundedness on $L^{2}\left(\mathbb{R}^{3}\right)$ and $L^{p}\left(\mathbb{R}^{3}\right)$. However, as the complexity of his method with the dimension increases, he did not reach a general result.

After years' exploration, the authors in [5] solved the question completely.

Theorem C (see [5]). Let $\theta=\left(\theta_{1}, \theta_{2}, \ldots, \theta_{n}\right) \in \mathbb{R}^{n}$ and $\Gamma_{\theta}(t)=$ $\left(\theta_{1}|t|^{p_{1}}, \theta_{2}|t|^{p_{2}}, \ldots, \theta_{n}|t|^{p_{n}}\right)$. Define $H_{n, \alpha, \beta}$ as

$$
H_{n, \alpha, \beta} f(x)=\int_{-1}^{1} f\left(x-\Gamma_{\theta}(t)\right) e^{i|t|^{-\beta}}|t|^{-1-\alpha} d t, \quad \beta>\alpha .
$$

If $p_{1}, p_{2}, \ldots, p_{n}, \alpha, \beta$ are all positive,

(1) $\left\|H_{n, \alpha, \beta} f\right\|_{L^{p}\left(\mathbb{R}^{n}\right)} \preceq\|f\|_{L^{p}\left(\mathbb{R}^{n}\right)}$, as long as $\beta>(n+$ $1) \alpha$ and $2 \beta /(2 \beta-(n+1) \alpha)<p<2 \beta /(n+1) \alpha$;

(2) $\left\|H_{n, \alpha, \beta} f\right\|_{L^{2}\left(\mathbb{R}^{n}\right)} \preceq\|f\|_{L^{2}\left(\mathbb{R}^{n}\right)}$, if $\beta=(n+1) \alpha$.

Further on, the authors [6] proved that if $p_{i}$ are mutually different, then

$$
\left\|H_{n, \alpha, \beta} f\right\|_{L^{2}\left(\mathbb{R}^{n}\right)} \preceq\|f\|_{L^{2}\left(\mathbb{R}^{n}\right)} \Longleftrightarrow \beta \geq(n+1) \alpha .
$$

In [5], it is showed that we only need to consider the part of $t \geq 0$, and $\Gamma_{\theta}(t)$ could be reduced to $\Gamma(t)=\left(t^{p_{1}}, t^{p_{2}}, \ldots, t^{p_{n}}\right)$. That is the operator which is given at the very start:

$$
H_{n, \alpha, \beta} f(x)=\int_{0}^{1} f(x-\Gamma(t)) e^{i t^{-\beta}} t^{-1-\alpha} \mathrm{d} t,
$$

and so is what we will discuss in the next section. Just under the bases of $[5,6]$, we probe into the boundedness of $H_{n, \alpha, \beta}$ on Sobolev spaces.

\section{Preliminary and Main Results}

As we know, smoothness is a crucial property of functions, and it is common to use high-ordered continuity to describe it. Yet an arbitrary function is not always differentiable. Due to this, Sobolev spaces are introduced to measure the differentiability of some more common functions. These spaces are widely used in both harmonic analysis and PDE.

There are several equivalent definitions of such spaces. Let us start with the classical definition. Firstly, we need to recall the concept of generalized derivatives.

Definition 1. Let $u \in \mathcal{S}^{\prime}$ and let $\alpha$ be multiple index. Define

$$
\left\langle\partial^{\alpha} u, f\right\rangle=(-1)^{|\alpha|}\left\langle u, \partial^{|\alpha|} f\right\rangle \text {. }
$$

If $u$ is a function, then $\partial^{\alpha} u$, the derivative of $u$, in the meaning of distribution, is called weak derivative.
Definition 2 (see [7]). Let $k$ be a nonnegative integer and $1<$ $p<\infty$. We can define the Sobolev spaces $L_{k}^{p}\left(\mathbb{R}^{n}\right)$ as follows:

$$
L_{k}^{p}\left(\mathbb{R}^{n}\right)=\left\{f \in L^{p}\left(\mathbb{R}^{n}\right)\left|\partial^{\alpha} f \in L^{p}\left(\mathbb{R}^{n}\right), \forall \alpha,\right| \alpha \mid \leq k\right\} .
$$

And the norm is given as

$$
\|f\|_{L_{k}^{p}\left(\mathbb{R}^{n}\right)}=\sum_{|\alpha| \leq k}\left\|\partial^{\alpha} f\right\|_{L^{p}\left(\mathbb{R}^{n}\right)},
$$

where $\partial^{(0, \ldots, 0)} f=f$.

It is easy to see that $L_{k}^{p}\left(\mathbb{R}^{n}\right)$ is a proper subspace of $L^{p}\left(\mathbb{R}^{n}\right)$. The indice $k$ characterizes the smoothness of the function spaces, and we have the following inclusion relations:

$$
L^{p}\left(\mathbb{R}^{n}\right) \supset L_{1}^{p}\left(\mathbb{R}^{n}\right) \supset L_{2}^{p}\left(\mathbb{R}^{n}\right) \supset L_{3}^{p}\left(\mathbb{R}^{n}\right) \supset \cdots
$$

In the above definition, $k$ should be an integer. Further on, we can extend the definitions, without assuming $k$ to be an integer.

Definition 3 (see [7]). Let $s$ be real and $1<p<\infty$. The inhomogeneous Sobolev spaces $L_{s}^{p}\left(\mathbb{R}^{n}\right)$ consisted of all the elements $u$ of $\mathcal{S}^{\prime}$, which satisfies the following property:

$$
\left(\left(1+|\xi|^{2}\right)^{s / 2} \widehat{u}\right) \smile \in L^{p}\left(\mathbb{R}^{n}\right)
$$

And the corresponding norm is given below:

$$
\|u\|_{L_{s}^{p}\left(\mathbb{R}^{n}\right)}=\left\|\left(\left(1+|\xi|^{2}\right)^{s / 2} \widehat{u}\right)-\right\|_{L^{p}\left(\mathbb{R}^{n}\right)} .
$$

For the definition, there are some observations:

(1) if $s=0, L_{s}^{p}\left(\mathbb{R}^{n}\right)=L^{p}\left(\mathbb{R}^{n}\right)$;

(2) for every $s, L_{s}^{p}\left(\mathbb{R}^{n}\right)$ is subset of $L^{p}\left(\mathbb{R}^{n}\right)$;

(3) if $s=k$ is a nonnegative integer, the two definitions coincide.

Along with inhomogeneous Sobolev spaces, we can give the definition of the homogeneous Sobolev spaces.

Definition 4 (see [7]). Let $s$ be a real number and $1<p<\infty$. We define homogeneous Sobolev spaces $\dot{L}_{s}^{p}\left(\mathbb{R}^{n}\right)$ as follows:

$$
\dot{L}_{s}^{p}\left(\mathbb{R}^{n}\right)=\left\{u \mid u \in \frac{\mathcal{S}^{\prime}\left(\mathbb{R}^{n}\right)}{\mathscr{P}},\left(|\xi|^{s} \widehat{u}\right)^{`} \in L^{p}\left(\mathbb{R}^{n}\right)\right\},
$$

and, for the distributions in $\dot{L}_{s}^{p}\left(\mathbb{R}^{n}\right)$, we can define

$$
\|u\|_{\dot{L}_{s}^{p}\left(\mathbb{R}^{n}\right)}=\left\|\left(|\cdot|^{s} \widehat{u}\right){ }^{-}\right\|_{L^{p}\left(\mathbb{R}^{n}\right)} .
$$

What should be noticed is that the elements of homogeneous Sobolev spaces $\dot{L}_{s}^{p}\left(\mathbb{R}^{n}\right)$ may not belong to $L^{p}\left(\mathbb{R}^{n}\right)$. Actually, these elements are equivalent classes of the temper distributions. For more details, please refer to chapter 6 of [7]. 
We also need the following Van der Corput Lemma, which is the most important lemma to estimate the oscillating integrals.

Van der Corput Lemma. Let $\psi$ and $\phi$ be smooth real functions in $(a, b)$, and $k \in \mathbb{N}$. If $\left|\psi^{(k)}(t)\right| \geq 1$ for all $t \in(a, b)$ and one of the two below conditions are satisfied: (1) $k=1, \psi^{\prime}(t)$ is monotone in $(a, b)$; (2) $k \geq 2$, then

$$
\left|\int_{a}^{b} e^{i \lambda \psi(t)} \phi(t) \mathrm{d} t\right| \leq C_{k} \lambda^{-1 / k}\left(|\phi(b)|+\int_{a}^{b}\left|\phi^{\prime}(t)\right| \mathrm{d} t\right) .
$$

The main results of the paper are as follows.

Theorem 5. For the operator $H_{n, \alpha, \beta}$, in the definition of $\Gamma_{\theta}(t)$, $p_{1}, p_{2}, \ldots, p_{n}, \alpha, \beta$ are all positive. If $\beta>(n+1) \alpha$ and $2 \beta /(2 \beta-$ $(n+1) \alpha)<p<2 \beta /(n+1) \alpha$, then

$$
\left\|H_{n, \alpha, \beta} f\right\|_{L_{s}^{p}\left(\mathbb{R}^{n}\right)} \preceq\|f\|_{L_{s}^{p}\left(\mathbb{R}^{n}\right)} .
$$

Theorem 6. For the operator $H_{n, \alpha, \beta}$, in the definition of $\Gamma_{\theta}(t)$, $p_{1}, p_{2}, \ldots, p_{n}, \alpha, \beta$ are all positive. If $\alpha<\beta(N+1 /(n+1))$ and $N$ is the biggest integer not more than $s$, then

$$
\left\|H_{n, \alpha, \beta} f\right\|_{L^{2}\left(\mathbb{R}^{n}\right)} \preceq\|f\|_{L_{s}^{2}\left(\mathbb{R}^{n}\right)} .
$$

\section{Proof of the Main Results}

Proof of Theorem 5. To deal with the singularity on the denominator of the operator $H_{n, \alpha, \beta}$, a dyadic decomposition is introduced.

Suppose $\Phi$ is a $C^{\infty}$ function, supported on $[1 / 2,2]$. By normalization, it can be assumed that

$$
\sum_{j=-\infty}^{+\infty} \Phi\left(2^{j} t\right) \equiv 1
$$

is true for all $t>0$. So we can decomposite $H_{n, \alpha, \beta}$ as follows:

$$
\begin{aligned}
H_{n, \alpha, \beta} f(x) & =\sum_{j=0}^{\infty} \int_{0}^{1} \Phi\left(2^{j} t\right) f(x-\Gamma(t)) e^{i t^{-\beta}} t^{-1-\alpha} \mathrm{d} t \\
& =\sum_{j=0}^{\infty} H_{j} f(x) .
\end{aligned}
$$

On account of the support of $\Phi$, we only need to consider the case where $j \geq 0$.

By Minkowski's inequality, it is easy to obtain the boundedness of $H_{j}$ on $L^{1}\left(\mathbb{R}^{n}\right)$ :

$$
\left\|H_{j} f\right\|_{L^{1}\left(\mathbb{R}^{n}\right)} \preceq 2^{j \alpha}\|f\|_{L^{1}\left(\mathbb{R}^{n}\right)} .
$$

Taking Fourier transform, we get the multiple form of $H_{j} f$ :

$$
\widehat{H_{j} f}(\xi)=m_{j}(\xi) \widehat{f}(\xi)
$$

where

$$
\begin{aligned}
m_{j}(\xi) & =m_{j}\left(\xi_{1}, \xi_{2}, \ldots, \xi_{n}\right) \\
& =\int_{0}^{1} \Phi\left(2^{j} t\right) t^{-1-\alpha} e^{i\left(t^{-\beta}-\sum_{k=1}^{n} \xi_{k} t^{p_{k}}\right)} \mathrm{d} t .
\end{aligned}
$$

In [5], the authors proved

$$
\left\|m_{j}\right\|_{L^{\infty}\left(\mathbb{R}^{n}\right)} \preceq 2^{j(\alpha-\beta /(n+1))} .
$$

Thus, by Plancherel's theorem, we have

$$
\begin{aligned}
\left\|H_{j} f\right\|_{L_{s}^{2}\left(\mathbb{R}^{n}\right)} & =\left\|\left(\left(1+|\xi|^{2}\right)^{s / 2} \widehat{H_{j} f}(\xi)\right)-\right\|_{L^{2}\left(\mathbb{R}^{n}\right)} \\
& =\left\|\left(1+|\xi|^{2}\right)^{s / 2} \widehat{H_{j} f}(\xi)\right\|_{L^{2}\left(\mathbb{R}^{n}\right)} \\
& =\left\|\left(1+|\xi|^{2}\right)^{s / 2} \widehat{f}(\xi) m_{j}(\xi)\right\|_{L^{2}\left(\mathbb{R}^{n}\right)} \\
& \preceq 2^{j(\alpha-\beta /(n+1))}\left\|\left(1+|\xi|^{2}\right)^{s / 2} \widehat{f}\right\|_{L^{2}\left(\mathbb{R}^{n}\right)} \\
& =2^{j(\alpha-\beta /(n+1))}\|f\|_{L_{s}^{2}\left(\mathbb{R}^{n}\right)} .
\end{aligned}
$$

So,

$$
\left\|H_{n, \alpha, \beta} f\right\|_{L_{s}^{2}\left(\mathbb{R}^{n}\right)} \preceq \sum_{j=0}^{\infty} 2^{j(\alpha-\beta /(n+1))}\|f\|_{L_{s}^{2}\left(\mathbb{R}^{n}\right)} .
$$

To make sure $H_{n, \alpha, \beta}$ is bounded on $L_{s}^{2}\left(\mathbb{R}^{n}\right)$ (for all $s$ ), it is only needed that $\beta>(n+1) \alpha$, which is the same as the requirement of the boundedness on $L^{2}\left(\mathbb{R}^{n}\right)$. Roughly speaking, the operators preserve the smoothness of the functions.

To get the boundedness on $L_{s}^{p}\left(\mathbb{R}^{n}\right)(p>1)$, we will use the interpolation between (25) and (29). It can be shown that

$$
\left\|H_{j}\right\|_{L_{2 s(1-1 / p)}^{p}\left(\mathbb{R}^{n}\right) \rightarrow L_{2 s(1-1 / p)}^{p}} \preceq 2^{j(\alpha-2 \beta(1-1 / p) /(n+1))} .
$$

As $s$ is arbitrary, it suffices to show that $\alpha-2 \beta(1-1 / p) /(n+1)<$ 0 ; that is,

$$
\frac{2 \beta}{2 \beta-(n+1) \alpha}<p \leq 2 \text {. }
$$

So $H_{n, \alpha, \beta}$ is bounded on $L_{s}^{p}\left(\mathbb{R}^{n}\right)$.

By duality argument, it is finally proved that if $\beta>(n+$ $1) \alpha$, then $H_{n, \alpha, \beta}$ is bounded on $L_{s}^{p}\left(\mathbb{R}^{n}\right)$, where $2 \beta /(2 \beta-(n+$ 1) $\alpha)<p<2 \beta /(n+1) \alpha$ and $s$ is arbitrary.

Theorem 5 indicates that the operator $H_{n, \alpha, \beta}$ can sustain the "smoothness" of functions. If what we care about is not the boundedness from Sobolev spaces to Sobolev spaces, but the boundedness from Sobolev spaces to $L^{p}$ spaces, then the lifting of the smoothness of $f$ can reduce the restriction of $\alpha$, $\beta$, which would be explained in the next theorem. 
Proof of Theorem 6. Here we will follow the notations and calculations in Theorem 5; that is,

$$
\begin{gathered}
H_{n, \alpha, \beta} f(x)=\sum_{j=0}^{\infty} H_{j} f(x) ; \quad \widehat{H_{j} f}(\xi)=m_{j}(\xi) \widehat{f}(\xi) ; \\
m_{j}(\xi)=\int_{0}^{1} \Phi\left(2^{j} t\right) t^{-1-\alpha} e^{i\left(t^{-\beta}-\sum_{k=1}^{n} \xi_{k} t^{p}\right)} \mathrm{d} t .
\end{gathered}
$$

Let $N$ be the largest integer, not exceeding $s$. For Sobolev spaces $L_{s}^{2}\left(\mathbb{R}^{n}\right)$, by Plancherel's theorem, when $s_{1}>s_{2}$,

$$
L_{s_{1}}^{2}\left(\mathbb{R}^{n}\right) \subset L_{s_{2}}^{2}\left(\mathbb{R}^{n}\right),
$$

and, for an element $f$ of $L_{s_{1}}^{2}$,

$$
\|f\|_{L_{s_{2}}^{2}\left(\mathbb{R}^{n}\right)}<\|f\|_{L_{s_{1}}^{2}\left(\mathbb{R}^{n}\right)} .
$$

The case $s_{1}=s, s_{2}=N$ will be used later.

We will make a more accurate estimation of $m_{j}$. Notice that $\Phi$ is a $C^{\infty}$ function, supported on $[1 / 2,2]$. By substitution of variables $2^{j} t \rightarrow t$, it is shown that

$$
m_{j}(\xi)=2^{j \alpha} \int_{0}^{\infty} \frac{\Phi(t)}{t^{1+\alpha}} e^{i\left(2^{j \beta} t^{-\beta}-\sum_{k=1}^{n} \xi_{k} 2^{-j p_{k} t_{k}}\right)} \mathrm{d} t=2^{j \alpha} m_{\alpha, \beta}
$$

where we extend the upper limit of the integral into infinity. Considering the support of $\Phi$ and $j \geq 0$, this extension will not make essential difference to the result.

In [5], the authors use Van der Corput Lemma and an elementary statement to prove

$$
\left\|m_{\alpha, \beta}\right\|_{L^{\infty}\left(\mathbb{R}^{n}\right)} \preceq 2^{-j \beta /(n+1)} .
$$

After thoughtful investigation of the proof in [5], it is unearthed that the part $\Phi(t) / t^{1+\alpha}$ will only contribute to the control constant in the inequality above, without any effect on the order of the index.

In the subsequent calculation, we will substitute the part $\Phi(t) / t^{1+\alpha}$ with notation $\Psi(t)$. Afterwards, $\Psi(t)$ always means a $C^{\infty}$ function, supported on $[1 / 2,2]$. With the process, $\Psi(t)$ will represent different functions, which will not do harm to the final result. That is, if $\Psi(t)$ is a $C^{\infty}$ function supported on $[1 / 2,2]$, then

$$
\begin{gathered}
\left\|\int_{0}^{\infty} \Psi(t) e^{i\left(2^{j \beta} t^{-\beta}-\sum_{k=1}^{n} \xi_{k} 2^{-j p_{k}} t^{p_{k}}\right)} \mathrm{d} t\right\|_{L^{\infty}\left(\mathbb{R}^{n}\right)} \preceq 2^{-j \beta / n+1}, \\
m_{\alpha, \beta}(\xi)=\frac{i}{2^{j \beta}} \int_{0}^{\infty} e^{-i\left(\sum_{k=1}^{n} \xi_{k} 2^{-j p_{k}} t^{p_{k}}\right)} \Psi(t) \mathrm{d} e^{i 2^{j \beta} t^{-\beta}}
\end{gathered}
$$

using integration by parts:

$$
\begin{aligned}
m_{\alpha, \beta}(\xi) & \\
= & \frac{-i}{2^{j \beta}} \int_{0}^{\infty} e^{i\left(2^{j \beta} t^{-\beta}-\sum_{k=1}^{n} \xi_{k} 2^{-j p_{k}} t^{p_{k}}\right)} \Psi(t) \mathrm{d} t \\
& +\sum_{k=1}^{n} \frac{\xi_{k} p_{k} 2^{-j p_{k}}}{2^{j \beta}} \int_{0}^{\infty} e^{i\left(2^{j \beta} t^{-\beta}-\sum_{k=1}^{n} \xi_{k} 2^{-j p_{k}} t^{p_{k}}\right)} \Psi(t) \mathrm{d} t .
\end{aligned}
$$

Notice that $\Psi$ indicates different functions in different places; still, they are all $C^{\infty}$ functions supported on $[1 / 2,2]$.

By (38), the absolute value of every integral above can be dominated by $2^{-j \beta /(n+1)}$. Along with Cauchy's inequality, we have

$$
\left|m_{\alpha, \beta}(\xi)\right| \preceq 2^{-j \beta /(n+1)} 2^{-j \beta}\left(1+|\xi|^{2}\right)^{1 / 2}
$$

Repeating integration by parts, it is suggested, for any $M$, that

$$
\left|m_{\alpha, \beta}(\xi)\right| \preceq 2^{-j \beta /(n+1)} 2^{-j M \beta}\left(1+|\xi|^{2}\right)^{M / 2} .
$$

So an estimation to the $L^{2}$ norm of $H_{j} f$ could be made. Recall that $N$ represents the largest integer not exceeding $s$ :

$$
\begin{aligned}
\left\|H_{j} f\right\|_{L^{2}\left(\mathbb{R}^{n}\right)} & =\left\|\widehat{H_{j} f}\right\|_{L^{2}\left(\mathbb{R}^{n}\right)} \\
& =\left\|m_{j} \widehat{f}\right\|_{L^{2}\left(\mathbb{R}^{n}\right)} \\
& \preceq 2^{j \alpha-j N \beta-j \beta /(n+1)}\left\|\left(1+|\xi|^{2}\right)^{N / 2} \widehat{f}\right\|_{L^{2}\left(\mathbb{R}^{n}\right)} \\
& =2^{j \alpha-j N \beta-j \beta /(n+1)}\|f\|_{L_{N}^{2}\left(\mathbb{R}^{n}\right)} \\
& \leq 2^{j \alpha-j N \beta-j \beta /(n+1)}\|f\|_{L_{s}^{2}\left(\mathbb{R}^{n}\right)^{.}}
\end{aligned}
$$

Further on, to guarantee $H_{n, \alpha, \beta}$ is bounded from $L_{s}^{2}\left(\mathbb{R}^{n}\right)$ to $L^{2}\left(\mathbb{R}^{n}\right)$, it is only needed that

$$
\alpha-N \beta-\frac{\beta}{n+1}<0 ;
$$

that is, $\alpha<\beta(N+1 /(n+1))$.

When $s=0, N=0$; that is, $\beta>(n+1) \alpha$, which is the result in [5].

\section{Conflict of Interests}

The authors declare that there is no conflict of interests regarding the publication of this paper.

\section{Acknowledgment}

This research was supported by PSF of Zhejiang province (BSH1302046).

\section{References}

[1] A. P. Calderón and A. Zygmund, “On singular integrals," American Journal of Mathematics, vol. 78, no. 2, pp. 289-309, 1956.

[2] E. B. Fabes and N. M. Rivière, "Singular integrals with mixed homogeneity," Studia Mathematica, vol. 27, no. 1, pp. 19-38, 1966.

[3] M. Zielinski, Highly oscillatory singular integrals along curves [Ph.D. dissertation], University of Wisconsin-Madison, Madison, Wis, USA, 1985.

[4] S. Chandarana, " $L^{p}$-bounds for hypersingular integral operators along curves," Pacific Journal of Mathematics, vol. 175, no. 2, pp. 389-416, 1996. 
[5] J. Chen, D. S. Fan, M. Wang, and X. R. Zhu, " $L^{p}$ bounds for oscillatory hyper-hilbert transform along curves," Proceedings of the American Mathematical Society, vol. 136, no. 9, pp. 3145-3153, 2008.

[6] J. C. Chen, D. S. Fan, and X. R. Zhu, "Sharp $L^{2}$ boundedness of the oscillatory hyper-Hilbert transform along curves," Acta Mathematica Sinica: English Series, vol. 26, no. 4, pp. 653-658, 2010.

[7] L. Grafakos, Classical and Modern Fourier Analysis, China Machine Press, Beijing, China, 2005. 


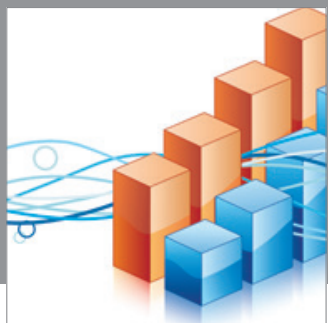

Advances in

Operations Research

mansans

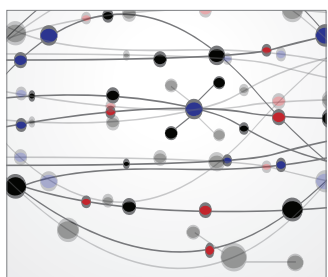

The Scientific World Journal
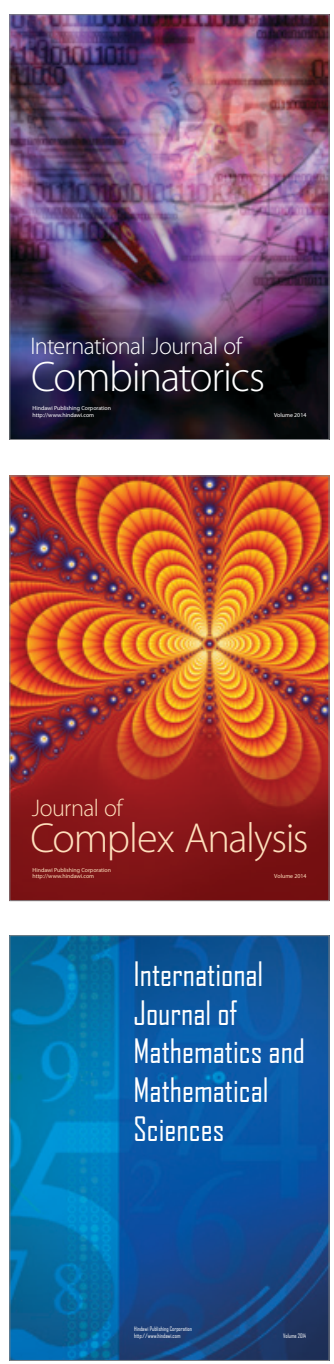
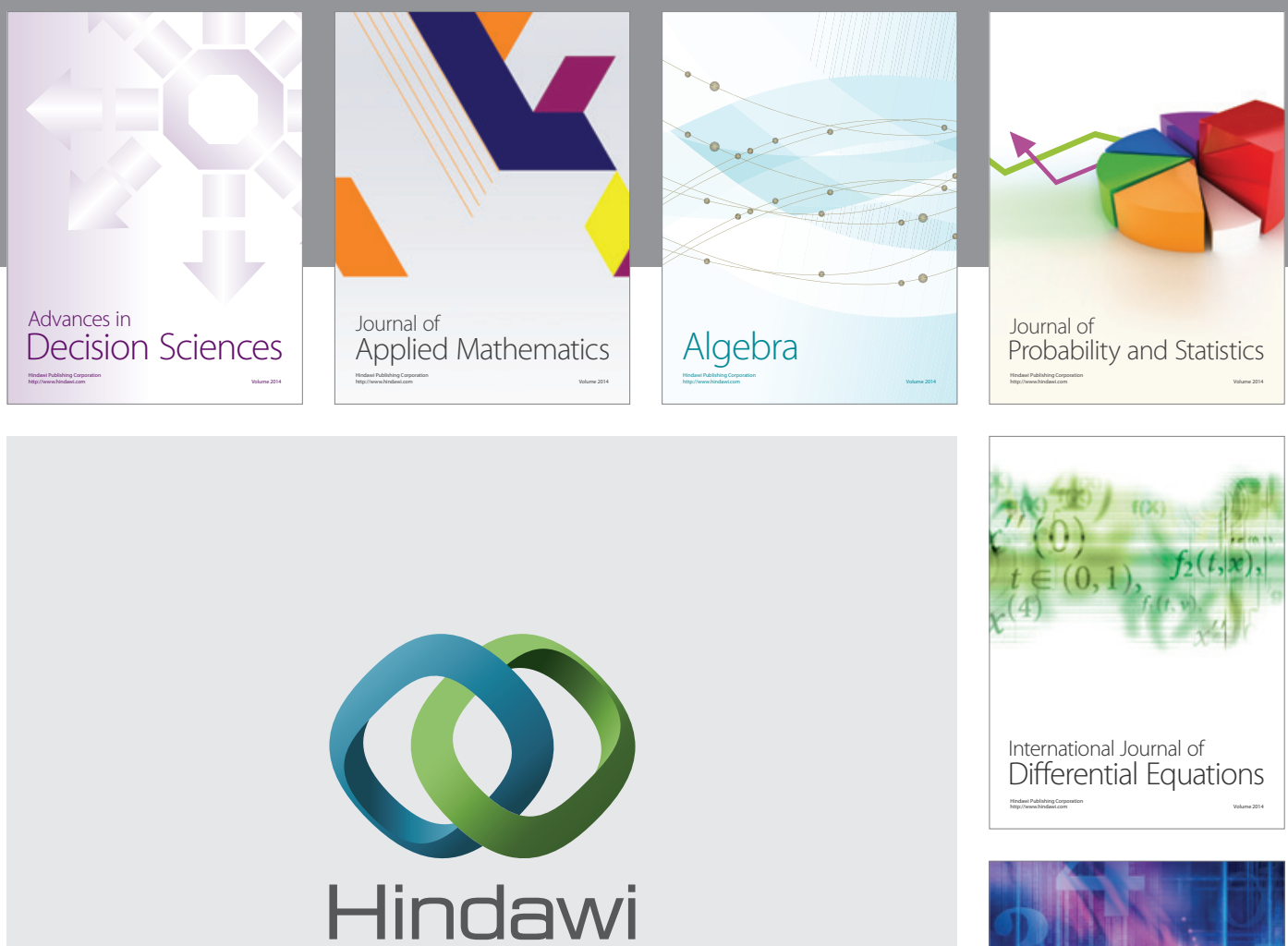

Submit your manuscripts at http://www.hindawi.com
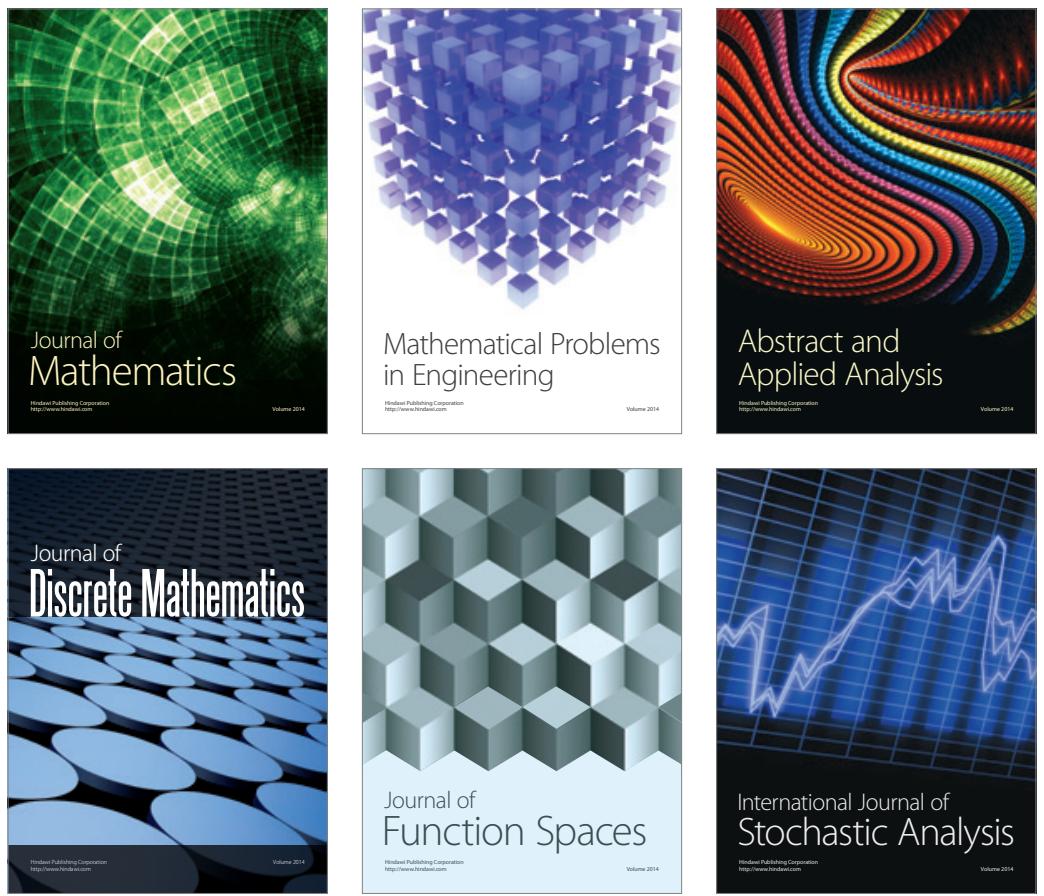

Journal of

Function Spaces

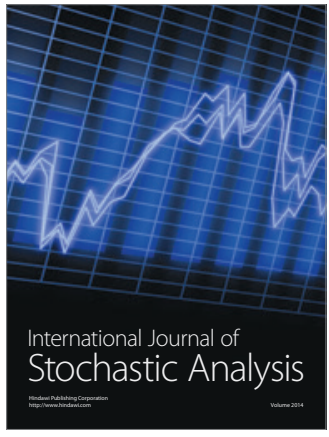

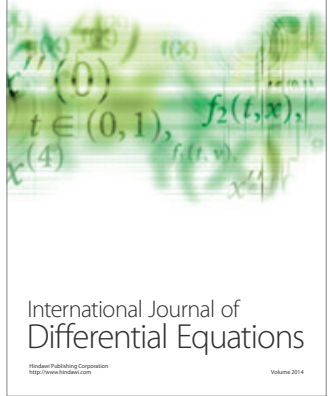
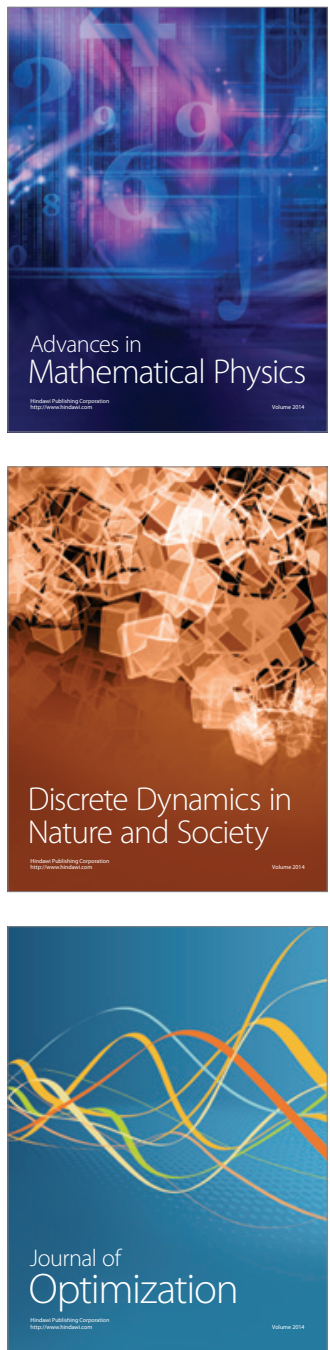